\title{
AFM nanoindentation detect elastic modulus of tongue squamous carcinoma cells with different metastatic potential
}

Running title: AFM nanoindentation detect elastic modulus of TSCC

Zhoulong Zhou ${ }^{2 \#}$, Chaoxu Zheng ${ }^{3 \#}$, Su Li, $\mathrm{PhD}^{4}$, Xiaofeng Zhou, $\mathrm{PhD}^{5}$, Zhonghua Liu ${ }^{1}$, Qianting He

${ }^{1}$, Ningning Zhang ${ }^{1}$, Alfonso Ngan, $\mathrm{PhD}^{2}$, Bin Tang, $\mathrm{PhD}^{2,6 \pi}$, Anxun Wang, $\mathrm{PhD}{ }^{1 \pi}$.

${ }^{1}$ Department of Oral and Maxillofacial Surgery, the First Affiliated Hospital, Sun Yat-Sen University, Guangzhou, China

${ }^{2}$ Department of Mechanical Engineering, University of Hong Kong, Hong Kong, China

${ }^{3}$ Department of General Surgery, the First Affiliated Hospital, Sun Yat-Sen University, Guangzhou, China

${ }^{4}$ Department of Medicine, Tumor Hospital, Sun Yat-Sen University, Guangzhou, China

${ }^{5}$ Department of Periodontics, College of Dentistry, University of Illinois at Chicago, Chicago, IL

${ }^{6}$ Department of Micro/Nano Materials and Devices, South University of Science and Technology of China, Shenzhen, China

\section{\#: These authors contributed equally to this work.}

\section{I:Corresponding author:}

Anxun Wang, Department of Oral and Maxillofacial Surgery, the First Affiliated Hospital, Sun Yat-Sen University, 58 Zhongshan Road II, Guangzhou, 510080, P.R. China; Phone: +86-0-13724896216; E-mail: anxunwang@yahoo.com.

Bin Tang, Department of Micro/Nano Materials and Devices, South University of Science and Technology of China, ShenZhen, China. E-mail: tangbinsci@gmail.com

Conflict of interest statement: No declared

Sources of support: This work was supported in part by grants from the National Nature Science Foundation of China (NSFC81072228, NSFC81272953,NSFC11202039), the Guangdong Natural Science Foundation (10151008901000093, S2011020002325), the International Cooperative Project of Science and Technology of Guangdong Province (1011420600001), 
and the Hong Kong General Research Fund (Project No. HKU 716908E). 


\begin{abstract}
Although significant advances have been made in the molecular mechanisms that influence tongue squamous cell carcinoma (TSCC) metastasis, less is known about the association between the biomechanical behavior of the TSCC cells and their metastasis. To this goal, atomic force microscope (AFM) nanoindentation via the rate-jump method was used to detect the elastic modulus of TSCC cells from patients and cell lines with different metastatic potential. We found that TSCC cells with higher metastatic potential showed a reduction in elastic modulus as compared to TSCC cells with lower metastatic potential; moreover, the decrease in elastic modulus was accompanied by changes in epithelial-mesenchymal transition (EMT) and cytoskeleton (F-actin and $\beta$-tubulin), a smaller nucleus size, and a large nucleus/cytoplasm ratio. The present findings demonstrate a close relationship between cellular elastic modulus and metastasis of TSCC. Also, the detection of elastic modulus by AFM nanoindentation via the rate-jump method can potentially be used as a method to grade the metastatic potential of TSCC.
\end{abstract}

\title{
Keywords
}

Tongue squamous cell carcinoma; elastic modulus; metastasis; atomic force microscopy; nanoindentation 


\section{Background}

Tongue squamous cell carcinoma (TSCC) is significantly more aggressive than other forms of oral squamous cell carcinomas (OSCC), and a propensity for rapid local lymph node (LN) metastasis. Improvement in patient survival requires a better understanding of tumor metastasis, which would allow aggressive tumors to be detected earlier in the disease process and targeted therapeutic interventions to be developed. Our previous studies revealed that the metastasis of TSCC may be related to many deregulated genes, such as SOD2, miR-138 and miR-222/221 [1-8]. In these studies we found that reductions in miR-138 are correlated with an enhanced metastatic potential and induce EMT (epithelial-mesenchymal transition) in TSCC cells. Similar to our studies, most of the current research regarding cancer metastasis has focused on biological capabilities such as molecular genetics and gene signaling, whereas the mechanical nature of the invasion process of the cancer cells has been ignored so far [9]. Indeed, the mechanical properties of living cells are strongly linked to the molecular alterations in the progression of cancer. Cumulative studies have revealed that the mechanical properties of individual cells (such as elastic and viscous) are important in the cell growth, motility and metastasis of cancer [10]. Malignant cancer cells are either less elastic (softer) or less viscous (less resistant to flow) than their normal counterparts, and metastatic cancer cells exhibit an even lower resistance to deformation than nonmetastatic cancer cells [11-13]. However, very few studies have focused on the effect of the elasticity of TSCC cells on their metastasis.

On the other hand, advances in experimental nano-biomechanics during the past two decades have enabled direct, real-time mechanical probing and the manipulation of single cells and molecules [10]. Commonly used experimental techniques include nanoindentation [14], micropipette aspiration [15] and microfluidic techniques [16]. Among these methods, nanoindentation is the most popular because quantitative measurement of the mechanical properties can be made directly without the need of carrying out time consuming post-experimental analyses. When nanoindentation is carried out on atomic force microscopes (AFM), the Hertzian model is commonly used to analyze the data to generate an elastic modulus of the sample [14, 17]. However, since the Hertzian model assumes the sample to be purely linear elastic, while the cytoplasm-membrane-cytoskeleton structure of a cell 
should not behave in a purely linear elastic manner, the Hertzian model is almost definitely a wrong description of the cell's nanoindenation response. In fact in such analyses of experimental data by the Hertzian model, the resultant elastic modulus estimates are often found to increase with the loading rate $[18,19]$. To solve this problem, our group has developed a rate-jump protocol for AFM nanoindentation which can produce much more reliable measurement of the elastic modulus of soft samples than the Hertzian model. This protocol has been found to yield elastic modulus that is independent of the rate-jump magnitude from soft samples [18-20].

It is well known that the mechanical properties of human cells are largely governed by their cytoskeleton [10]. During the descent of a cell from a mature, differentiated state to a highly mitotic and motile cancerous state, the cytoskeleton regresses from an ordered and fairly stiff structure to a more irregular and compliant state [21,22]. Furthermore, some other factors, such as EMT [23], the overall cell size, the nucleus size [24], and chromatin organization [25], are all found to affect the cell's mechanical behavior. However, exactly how these cellular structural factors influence the mechanical properties of cell remains quite ambiguous.

To investigate the relationship between the elastic modulus and metastatic potential of TSCC, the elastic modulus of cancer cells from cell lines with different metastatic potentials and primary cell samples from TSCC patients were examined using AFM nanoindentation via the above-mentioned rate-jump method [18, 19]. Moreover, various cellular structural factors such as the cytoskeleton structure, EMT, nucleus size, etc. were also investigated with various imagining techniques, so as to explore the structure-mechanical property relationship of different types of tongue cancer cells with different metastatic potential. 


\section{Methods}

\section{Primary cell culture}

Archived tissue samples for primary cell cultures from four TSCC cases were used in the present study. Clinical characterizations are summarized in Table S1. All of the patients underwent curative surgery and did not receive any adjuvant therapy prior to surgery, and all of the experiments with primary samples were performed within a few days after surgery. The present study was approved by the Ethical Committee of the First Affiliated Hospital, Sun Yat-Sen University. Primary cell cultures were maintained as previously described [22]. Briefly, TSCC tissues were disinfected with Betaisodona (Mundipharma, Limburg, Germany), rinsed twice in PBS, minced and placed in $2.5 \mathrm{mg} / \mathrm{ml}$ Dispase II (Roche, Mannheim, Germany) in DMEM for 18 to $24 \mathrm{~h}$ at $4{ }^{\circ} \mathrm{C}$. Subsequently, tissues were incubated in $0.25 \%$ trypsin/EDTA for 5 min. Trypsin activity was stopped with DMEM containing $10 \%$ fetal bovine serum (FBS). The suspensions were centrifuged, resuspended in DMEM/F12 (containing 10\% FBS, 1,000 units $/ \mathrm{ml}$ penicillin and $500 \mu \mathrm{g} / \mathrm{ml}$ streptomycin), and cultured at $37^{\circ} \mathrm{C}$ with $5 \% \mathrm{CO}_{2}$.

\section{Cell culture}

UM1 and UM2 are paired TSCC cell lines with different metastatic potentials that were previously established from a single patient [26]. Another two paired cell lines with different metastatic potentials (UM1/Control and UM1/miR-138, 1386Ln/Control and 1386Ln/miR-138) were also used in this study [6]. These cell lines were stable transfected cells with control mimic or miR-138, respectively. 1386Ln is a cell line generated from lymph node metastatic disease from hypopharynx squamous cell carcinoma [6]. All the cells were maintained in DMEM/F12 containing 10\% FBS, 1,000 units/ml of penicillin and $500 \mu \mathrm{g} / \mathrm{ml}$ of streptomycin in a $37^{\circ} \mathrm{C}$ incubator with $5 \% \mathrm{CO}_{2}$.

\section{Wound healing assay}

Wound healing assay experiments were performed as previously described [27]. Images were taken at time points $0 \mathrm{~h}$ and $24 \mathrm{~h}$ post-wounding using a Nikon Diaphot TMD inverted microscope (4x). The relative distance traveled by the leading edge from $0 \mathrm{~h}$ to $24 \mathrm{~h}$ was assessed using Photoshop 7.0 software $(n=6)$.

Transwell invasion assay 
Transwell invasion assay experiments were performed as previously described [27]. Briefly, Biocoat Matrigel invasion chamber inserts (BD Biosciences, NJ, USA) were equilibrated for $2 \mathrm{~h}$ at $37{ }^{\circ} \mathrm{C}$ in serum-free medium. Cells were seeded in serum-free medium in the upper chamber and allowed to invade through the Matrigel to the lower chamber for $24 \mathrm{~h}$. Cells on the bottom surface of the filter were rinsed and permeabilized with $1 \%$ Triton X-100 in PBS for 20 min, stained with DAPI in the dark and visualized under a fluorescent microscope. Three random fields were captured at a magnification of $10 \times(n=3)$. The number of cells on the bottom surface was compared between groups.

\section{Western Blot Analysis}

Western blot analysis was performed as previously described [28] using antibodies specific to E-cadherin, vimentin (Cell Signaling Technology, Beverly, MA, USA) and GAPDH (Sigma-Aldrich, MO, USA).

\section{Immunohistochemical analysis}

Immunohistochemistry was performed according to previous described [29] to detect the EMT markers (E-cadherin and vimentin) using a commercially available kit (Invitrogen, Carlsbad, CA). The relative intensities of the completed immunohistochemical reactions were evaluated by 3 independently trained observers who were blinded to the clinical data. Image-Pro Plus v6.0 software (Media Cybernetics, USA) was used to score the relative intensities.

\section{Scanning electron microscopy}

SEM was performed to investigate alterations in the cellular morphology. Briefly, cells were plated onto $14 \mathrm{~mm}$ diameter coverslips and placed in a 24-well plate for $24 \mathrm{~h}$. Cells were then washed with PBS buffer and fixed with $2.5 \%$ glutaraldehyde in $0.1 \mathrm{M}$ cacodylate buffer for $2 \mathrm{~h}$ at $4^{\circ} \mathrm{C}$. After washing with cacodylate buffer with $0.1 \mathrm{M}$ sucrose, cells were dehydrated for $5 \mathrm{~min}$ in an increasing series of ethanol solutions $(30 \%, 50 \%, 75 \%, 90 \%$ and $100 \%)$. The samples were dried in a Critical Point Dryer and coated with gold prior to SEM scanning (Hitachi S4800 FEG SEM, Japan).

\section{Nucleus size and nucleus/cytoplasm (N/C) ratio measurement}

The nucleus size and N/C ratio measurements were performed using immunofluorescence imaging 
[30]. Briefly, cells were fixed with $2.5 \%$ glutaraldehyde solution and permeabilized with $0.1 \%$ Triton X-100 in PBS. After a PBS wash, the cells were stained with anti- $\beta$-tubulin-Cy3 (Sigma-Aldrich, MO, USA) for cytoplasmic observation and Hoechst $33342(1 \mu \mathrm{g} / \mathrm{mL}$ in PBS, Sigma-Aldrich, MO, USA) for nuclear observation. The cells were then washed with PBS and observed under a fluorescent microscope. The emission wavelengths for the Hoechst 33342 and Cy3 $\beta$-tubulin are 330-380 nm and $510-560 \mathrm{~nm}$, respectively. The ImageJ (v. 1.45s) software by the National Institute of Health (NIH) was used to measure the areas of the cells and their nuclei, and the N/C ratio data were calculated with the following equation [31],

$$
N / C=\frac{\text { Area }_{\text {nucleus }}}{\text { Area }_{\text {cell }}-\text { Area }_{\text {nucleus }}}
$$

\section{Immunofluorescence analysis}

Cytoskeletal organization (F-actin and $\beta$-tubulin) was performed using the direct immunofluorescence method [30]. The filamentous actin (F-actin) was stained with a phalloidin-fluorescein isothiocyanate (phalloidin-FITC) conjugate solution (Sigma-Aldrich, MO, USA), and the $\beta$-tubulin was stained with anti- $\beta$-tubulin-Cy3. The slides were examined using a fluorescence microscope (Leica, Germany). Representative images of the cells were captured at a magnification of $400 \times$ and in the same exposure times of 200 milliseconds. The emission wavelength for the FITC-phalloidin and Cy3 $\beta$-tubulin are $450-490 \mathrm{~nm}$ and $510-560 \mathrm{~nm}$, respectively. The mean intensity of F-actin and $\beta$-tubulin inside each entire cell was measured using ImageJ. In this method, the image was first converted into 8-bit format, and was then duplicated and used to determine the corresponding measured regions. A threshold function was applied to highlight the structure in the copy image and the resultant image was then converted into a binary image using the "binary" function of ImageJ. This image was then converted back to the original 8-bit format, and the mean intensity of the filaments was obtained by the function "analyze particles" in the software. In each group, the mean intensity of the filaments was obtained from 10 cells, and the corresponding mean intensities of different groups were compared, and in the Results section below, a typical image from each group is shown. 


\section{Atomic force microscopy nanoindentation}

Nanoindentation experiments were performed in an AFM (JPK NanoWizard II, Germany) to measure the cellular elastic modulus as previously described [19, 20]. A silicon nitride cantilever-tip (CSG01, supplied by NT-MDT Company, Russia) was used, and the end of the tip was cut off using focused ion-beam (FIB) milling (FEI Quanta 200 3D FIB/SEM) to form a flat-ended cylindrical tip with diameter $1.01 \mu \mathrm{m}$ as shown in our previous study [20]. The choice of the flat-ended tip geometry with a cylindrical shape here ensures that the tip-cell contact area is a constant during the nanoindentation test, and this greatly simplifies the analysis [32]. The cantilever sensitivity was calibrated by indenting on the glass substrate in the presence of DMEM cell culture medium, and the spring constant of the tip was calibrated to be $0.037 \mathrm{~N} / \mathrm{m}$ using the thermal fluctuation method prior to AFM nanoindentation. The cells were cultured on glass coverslips and placed on a Biocell ${ }^{\mathrm{TM}}$ holder (JPK, Germany), which ensured a constant $37^{\circ} \mathrm{C}$ environment for the cells during the entire AFM nanoindentation assay. AFM nanoindentation was performed by controlling the movement of the clamping base of the AFM cantilever-tip via a PZT scanner while the sample sat on a stationary base, and the deflection of the AFM cantilever at the tip's end was recorded by a position-sensitive photo-diode detector.

The indentation schedule employed in this study is shown in Figure 1A and 1B. Each cell was mechanically probed by AFM at its most bulging part, where the nucleus was likely to be, as shown in Figure S1. The cell is not homogeneous, and therefore the values of elastic modulus may change significantly at different positions of the cell. In this case, indenting on the most bulging part of cell with the help of microscopic observation should largely reduce measurement errors [33, 34]. Moreover, indenting on this location will ensure that the membrane-cytoskeleton-nucleus response of the cell to be measured which is more pertinent to metastasis through narrow openings. The indentation depth was chosen to be at least $1 \mu \mathrm{m}$ in order to better simulate deformations which occur physiologically as described by $\mathrm{Xu}$ et al [35]. Instead of running the test at a constant displacement rate as in the Hertzian method, in the rate jump method, a step change in the displacement rate is imposed, which results in a step change in the load rate. Theoretical considerations as previous described have shown that the relationship between these two step changes yields an elastic modulus 
which is intrinsic of any power-law viscoelastic material [36]. As is shown in our previous study, the Hertzian method gives an apparent elastic modulus which increases with the loading rate, but the rate-jump method gives elastic modulus values which are independent of the rate-jump magnitude [19]. In the present study, each cell was just indented once, and 30 cells were randomly selected to be indented in each sample group for further statistical analysis.

In the rate-jump protocol, the elastic modulus of the sample is given by [19]

$$
\Delta \dot{P}=2 a\left(\frac{E}{1-v^{2}}\right) \Delta \dot{h}
$$

where $h$ and $P$ are the indentation depth and indentation force, and $a, E$ and $v$ are the radius of the cylindrical tip's end (0.505 micron in the present study), the sample's intrinsic Young's modulus and Poisson's ratio, respectively. In Eq. (2), $\Delta \dot{P}$ and $\Delta \dot{h}$ are jumps in the rates of $P$ and $h$ across the unloading point, and in the AFM used, the indentation force $P$ is evaluated as $P=k(\delta-h)$ where $k$ is the spring constant of the AFM cantilever, $\delta$ is the programmed movement of the clamp base of the cantilever, and $h$ is the deflection of the AFM tip as detected by the photo-diode detector (see ref. [19] for details). As explained in detail elsewhere [18, 20, 36], the rate-jump method involving eqn. (2) assumes the tested sample to be viscoelastic, and the elastic modulus $E /\left(1-v^{2}\right)$ returned from eqn. (2) is the resultant of all the elastic spring elements in the viscoelastic constitutive law of the sample while the viscous dashpot elements, which can be nonlinear in general, do not play any role. Eqn. (2) is the result of a subtraction between the effects of the constitutive law just before and just after the rate-jump point [36], and the viscous dashpots are immaterial here because their deformations are continuous across the rate jump and are therefore subtracted out, i.e. they do not react to the rate jump. Only the elastic springs react to the rate jump and this is why eqn. (2) contains only the net elastic modulus although the sample is viscoelastic. In the event if the spring and dashpot elements are time or load-history dependent, as would be the case for large deformations when the cytoskeleton, for example, becomes significantly modified, then the $E /\left(1-v^{2}\right)$ returned from eqn. (2) will be the instantaneous value at the moment when the rate jump is applied [36]. The validity of this rate-jump 
method in general has been verified in a wide range of soft materials on different mechanical testing platforms, including depth-sensing indentation on bone tissues, polymers and soft metals $[18,37,38]$, micro-plate compression on stem-cell inserted collagen micro-masses for tissue engineering purposes [39], AFM nanoindentation of cells and soft polymers [19,20, 39], as well as macroscopic uniaxial tensile testing on polymers [40]. In the results below, the values of the Young modulus $E$ are reported and these were calculated from the measured reduced moduli $E /\left(1-v^{2}\right)$ by assuming the Poisson ratio $v$ of the cells to be 0.5 .

\section{Statistics}

The data were analyzed with Student's $t$ test to determine the significance between two variables or one-way analysis of variance (ANOVA) to calculate the significance when there were more than two variables. Associations between elastic modulus and cell migration and invasion were tested using Pearson's product-moment correlation and Spearman's rank correlation. 


\section{Results}

\section{Elastic modulus of TSCCs with different metastatic potentials}

Among the TSCC cases (Table S1), Case 3 had LN metastasis and recurrence after one month and died six months postsurgery; however, Cases 1, 2 and 4 did not have LN metastasis, and no recurrence occurred during the 10-18 months of follow-up. Using the wound healing and transwell assays, we found that UM1 cells and UM1/Control cells had a higher migratory and invasive ability (i.e. higher metastatic potential) than UM2 cells and UM1/miR-138 cells, respectively (Figure 2). Similarly, primary cells obtained from Case 3 had the highest metastatic potential than those obtained from the other three cases (Figure 2). In another paired OSCC cell line, 1386Ln/control cells exhibited significantly elevated metastatic potential when compared to $386 \mathrm{Ln} / \mathrm{miR}-138$ cells (Figure S2).

Atomic force microscope nanoindentation via the rate-jump method was used to detect the elastic modulus of these TSCC cells with different metastatic potentials. As shown in Figure 1C, Table 1 and Table S2, UM1 cells, UM1/Control cells and 1386Ln/Control cells (with higher metastatic potential) had significantly lower elastic modulus values when compared to UM2, UM1/miR-138 and 1386Ln/miR-138 cells (with lower metastatic potential), respectively. Primary cells obtained from Case 3 also exhibited significantly lower elastic modulus when compared to primary cells obtained from the other three cases. The histograms for each sample were provided in supplemental Figure S3, in which the distribution of elastic modulus values for the less invasive cells showed a normal distribution. The relationship between cellular elastic modulus and metastatic potential was further examined and indicated a strong association between elastic modulus and metastatic potential as shown in Table S3. These results revealed that cells with higher metastatic potential had lower elastic modulus values than cells with lower metastatic potential, which means that they were softer or more deformable.

\section{The relationship between EMT and elastic modulus of TSCC}

To investigate the relationship between elastic modulus and EMT, TSCCs with different metastatic potential were observed under SEM and the expression of EMT markers (E-cadherin and vimentin) was also assessed. Figure 3 and Figure S4A show that the primary cells obtained from Case 3, UM1 
cells, UM1/Control cells and 1386Ln/Control cells (all with lower elastic modulus and higher metastatic potential) were all spread out and had a fibroblastic morphology. On the other hand, primary cells obtained from the other three cases, UM2 cells, UM1/miR-138 cells and 1386Ln/miR-138 cells (all with higher elastic modulus and lower metastatic potential) were more adherent to one another showing promoted formation of cell clusters. More surface protrusions, microvilli and filopodia-like processes were found in cells with lower elastic modulus and higher metastatic potential.

Moreover, as shown in Figure 4, primary cells obtained from Case 3, UM1 cells and UM1/Control cells (with lower elastic modulus and higher metastatic potential) had higher expression levels of vimentin and lower expression levels of E-cadherin as compared with primary cells obtained from the other three cases, UM2 cells and UM1/miR-138 cells (with higher elastic modulus and lower metastatic potential), respectively. These observations are in agreement with the hypothesis that EMT may be related to the elastic modulus and metastatic potential.

\section{The relationship between Cytoskeleton and elastic modulus of TSCC}

As shown in Figures 5, 6, and Figure S4B, the F-actin cytoskeleton in TSCCs features a dense cortical layer underneath the plasma membrane and an isotropic network throughout the cell body. $\beta$-tubulin was localized in the space between the nucleus and the cell membrane. F-actin and $\beta$-tubulin intensity profiles in the primary cells obtained from Cases 1, 2 and 4, UM2 cells, UM1/miR-138 cells and 1386Ln/miR-138 cells (with higher elastic modulus and lower metastatic potential) were significantly higher than those in primary cells obtained from Case 3, UM1 cells, UM1/Control cells and 1386Ln/Control cells (with lower elastic modulus and higher metastatic potential), respectively. Moreover, UM2 cells, UM1/miR-138 cells and 1386Ln/miR-138 cells had a thick cortical layer of F-actin and a thick nuclear layer of $\beta$-tubulin. These resluts imply that lower elastic modulus in TSCC cells with higher metastatic potential are related to less organization and decreased intensity of F-actin and $\beta$-tubulin.

\section{The relationship between nucleus size and elastic modulus of TSCC}

As shown in Table 2 and Table S2, primary cells obtained from Case 3, UM1 cells, UM1/Control cells 
and 1386Ln/Control cells (with lower elastic modulus and higher metastatic potential) had significantly smaller nucleus sizes and higher N/C ratios than primary cells obtained from the other three cases, UM2 cells, UM1/miR-138 cells and 1386Ln/miR-138 cells (with higher elastic modulus and lower metastatic potential), respectively. Thus, the nucleus size and the N/C ratio may lead to a reduction in the elastic modulus and an increase in metastatic potential. 


\section{Discussion}

The elastic modulus is an inverse measure of the deformability or compliance of individual cells and is therefore important in the initiation of cancer [10]. Many studies have shown that malignant tumor cells display either lower elastic modulus (softer) and/or lower viscosity (less resistant to flow) [13, 21, 41, 42]. Although Remmerbach et al. recently showed that both oral epithelial cell lines and primary cancer cells have an increased deformability compared with their normal counterparts [22], not many studies have focused on oral cancer. Recently researchers also found that mechanical properties are a critical determinant for the efficiency of cancer cell invasion [11, 21, 30, 41, 42]. Swaminathan et al. found that cancer cells with the highest migratory and invasive potential are five times less stiff (with lower elastic modulus) than cells with the lowest migration and invasive potential [11]. In the present study on tongue cancer cells, we revealed that, just like other types of cancer, cells with lower metastatic potential from TSCC cell lines and patients had significantly higher elastic modulus (i.e., less deformable) than those of cells with higher metastatic potential. The elastic modulus of tongue cancer cells with lower metastatic potential is only around twice of that of cells with higher metastatic potential, which means that the difference between them is less significant than those reported in previous studies [11]. It is believed that this is due to the individual differences among different types of cancer cells. It should also be noted that, based on the elastic modulus measured in this work, the difference between elastic modulus of tongue cancer cells with high metastatic and low metastatic potential is less significant than those of other types of cancers, and therefore a protocol for accurate measurement of the elastic modulus of cancer cells is necessary for evaluating the metastatic potential. Thus, these findings suggest that cellular elastic modulus may be related to the metastasis of TSCC and can be used as a cell marker and a diagnostic parameter for TSCC metastasis. Moreover, the present study also shows that the rate-jump method for AFM nanoindentation can be used as a standard protocol for measuring the elastic modulus of living cells, since the measured values are intrinsic properties of the cells.

Tumor metastasis is the dominant cause of death in patients with TSCC. EMT is a critical step in the process of metastasis, which leads to cancer spread and treatment failure. Recently, studies from our 
group and other groups have demonstrated that EMT is important in determining the biological behaviors and clinical prognosis of tongue cancer [5, 43]. For example, Sakamoto found that the development of delayed neck metastasis in stage I/II TSCC was closely related to the induction of EMT in primary tumor cells [43]. Our previous studies have suggested that downregulation of miR-138 is associated with mesenchymal-like cell morphology and enhanced cell migration and invasion [3-8]. Besides, EMT-associated changes were also found to influence cellular mechanics [44]. Buckley et al, found that alveolar epithelial cells (A549) with TGF- $\beta 1$ stimulation gave rise to a significant increase in stiffness and induced EMT changes [44]. In the present study, we also found that TSCC cells with lower elastic modulus and higher metastatic potential had EMT changes. These observations revealed that EMT might lead to a decrease in elastic modulus and an increase in the metastatic potential of TSCC.

The cytoskeleton is the structural framework that predominantly shapes a cell and provides its mechanical rigidity. According to the tensegrity theory, a cell can be viewed as a tensional structure in which actin filaments bear tensile loads and microtubules bear compression loads [30]. Among the different cytoskeletal filaments, F-actin provides the highest resistance to deformation [10]. Changes in the cytoskeleton would therefore reflect the overall mechanical properties of the cell [21, 24, 45-47]. Compared with nonmetastatic cancer cells, Guck et al. found that the increased deformability (decrease in elastic modulus) of cancer cells with metastatic competence appears to be accompanied by a reduction in the amount of F-actin [21]. In the present study, we observed differences in the cell cytoskeleton (F-actin and $\beta$-tubulin) that were accompanied with changes in cell metastatic potential and elastic modulus. In the cells with lower elastic modulus and higher metastatic potential, the cytoskeleton was reduced and less organized, with lower levels of F-actin gathered underneath the plasma membrane and decreased levels of $\beta$-tubulin organized around the nucleus. These observations confirmed that changes in the cytoskeleton may regulate the cellular elastic modulus and induce cell motility, invasion and metastasis.

Directed-cell migration, which often requires nuclear reshaping to allow passage of cells through narrow openings, is dependent not only on changes in cytoskeletal elements but also on global 
chromatin condensation $[25,48]$. Rolli et al. found that the diameters of the nuclei of pancreatic epithelial cancer cells (Panc-1) are reduced by 12\% after sphingosylphosphorylcholine treatment, which can enhance the invasive behavior and deformability of Panc-1 [24]. Stroka et al. also found that increased cell stiffness correlates with an increase in cell area [49]. Similar to these studies, we demonstrated that TSCC cells with higher metastatic potential had smaller nuclei and higher N/C ratios than cells with lower metastatic potential. Thus the small nuclei may decrease the overall elastic stiffness of the cells and allowed them to pass through narrow openings, and this corresponds to an increased invasion of TSCC cells.

From the above, the present findings revealed a close relationship between cellular elastic modulus and the metastatic potential of TSCC cells. The EMT, cytoskeleton, nucleus size and nucleus/cytoplasm ratio are all factors contributing to a change of the cellular elastic modulus. Combined with our previous studies [3-8], we hypothesize that TSCC cells with higher metastatic potential undergo a series of molecular changes, such as deregulation of miR-138, then induce EMT and cytoskeleton disorganization, modulate the nucleus size and nucleus/cytoplasm ratio, and finally have their cellular elastic modulus reduced and metastasis mediated. Thus, the cellular elastic modulus may be used to grade the metastatic potential of TSCC and as a diagnostic parameter for TSCC metastasis. In addition, since the present rate-jump method of AFM nanoindentation takes only minutes rather than hours to complete as in invasion assays, this is an efficient and reliable approach for classifying cancer cells to enable broad screening of suspicious lesions with metastasis of TSCC.

To conclude, the significance of this work can be summarized as follows. 1) We used a new method (rate-jump AFM nanoindentation) to measure the elastic modulus of cancer cells in which the viscous effects can be eliminated giving rise to reliable and intrinsic measurements of the elastic modulus. 2) Although tongue squamous cell carcinoma (TSCC) is significantly more aggressive than other forms of oral squamous cell carcinomas, how metastasis is linked to cellular biomechanics is basically unknown for this type of carcinoma. Using both TSCC cell lines and TSCC cells from patients with different metastatic potential, we found a close relationship between the elastic modulus and metastatic potential of TSCC cells. 3) With the efficient rate-jump AFM nanoindentation method, we 
demonstrated that the cellular elastic modulus can be used as a diagnostic parameter for grading the metastatic potential of TSCC. 4) By indenting on the nucleus location of the cells, we showed that the cellular elastic modulus (and hence metastatic potential) of TSCC cells are related to the filament organization in the cytoskeleton, the nucleus size and also the nucleus/cytoplasm ratio. 


\section{REFERENCES:}

1. Ye H, Wang A, Lee BS, Yu T, Sheng S, Peng T, et al. Proteomic based identification of manganese superoxide dismutase 2 (SOD2) as a metastasis marker for oral squamous cell carcinoma. Cancer Genomics Proteomics 2008; 5:85-94.

2. Liu X, Yu J, Jiang L, Wang A, Shi F, Ye H, et al. MicroRNA-222 regulates cell invasion by targeting matrix metalloproteinase 1 (MMP1) and manganese superoxide dismutase 2 (SOD2) in tongue squamous cell carcinoma cell lines. Cancer Genomics Proteomics 2009; 6:131-139.

3. Jiang L, Dai Y, Liu X, Wang C, Wang A, Chen Z, et al. Identification and experimental validation of $G$ protein alpha inhibiting activity polypeptide 2 (GNAI2) as a microRNA-138 target in tongue squamous cell carcinoma. Hum Genet 2011; 129:189-197.

4. Jiang L, Liu X, Kolokythas A, Yu J, Wang A, Heidbreder CE, et al. Downregulation of the Rho GTPase signaling pathway is involved in the microRNA-138-mediated inhibition of cell migration and invasion in tongue squamous cell carcinoma. Int J Cancer 2010; 127:505-512.

5. Liu X, Wang C, Chen Z, Jin Y, Wang Y, Kolokythas A, et al. MicroRNA-138 suppresses epithelial-mesenchymal transition in squamous cell carcinoma cell lines. Biochem J 2011; 440:23-31.

6. Liu X, Jiang L, Wang A, Yu J, Shi F, Zhou X. MicroRNA-138 suppresses invasion and promotes apoptosis in head and neck squamous cell carcinoma cell lines. Cancer Lett 2009; 286:217-222.

7. Jin Y, Wang C, Liu X, Mu W, Chen Z, Yu D, et al. Molecular characterization of the microRNA-138-Fos-like antigen 1 (FOSL1) regulatory module in squamous cell carcinoma. J Biol Chem 2011; 286:40104-9.

8. Wang C, Liu X, Chen Z, Huang H, Jin Y, Kolokythas A, et al. Polycomb group protein EZH2-mediated E-cadherin repression promotes metastasis of oral tongue squamous cell carcinoma. Mol Carcinog 2011, doi: 10.1002/mc.21848.

9. Shoushtari AN, Szmulewitz RZ, Rinker-Schaeffer CW. Metastasis-suppressor genes in clinical 
practice: lost in translation? Nat Rev Clin Oncol 2011; 8:333-342.

10. Suresh S. Biomechanics and biophysics of cancer cells. Acta Biomater 2007; 3:413-438.

11. Swaminathan V, Mythreye K, O'Brien ET, Berchuck A, Blobe GC, Superfine R. Mechanical stiffness grades metastatic potential in patient tumor cells and in cancer cell lines. Cancer Res 2011; 71:5075-5080.

12. Cross SE, Jin YS, Tondre J, Wong R, Rao J, Gimzewski JK. AFM-based analysis of human metastatic cancer cells. Nanotechnology 2008; 19:384003.

13. Suresh S. Nanomedicine: elastic clues in cancer detection. Nat Nanotechnol 2007; 2:748-749.

14. Lekka M, Laidler P. Applicability of AFM in cancer detection. Nat Nanotechnol 2009; 4:72.

15. Chen K, Li D, Jiang YH, Yao WJ, Wang XJ, Wei XC, et al. Influence of expressed TRAIL on biophysical properties of the human leukemic cell line Jurkat. Cell Res 2004; 14:161-168.

16. Song JW, Cavnar SP, Walker AC, Luker KE, Gupta M, Tung YC, et al. Microfluidic endothelium for studying the intravascular adhesion of metastatic breast cancer cells. PLoS One 2009; 4:e5756.

17. Ngan AHW, Tang B. Viscoelastic effects during unloading in depth-sensing indentation. Journal of Materials Research 2002; 17:2604-2610.

18. Tang B, Ngan AHW. A rate-jump method for characterization of soft tissues using nanoindentation techniques. Soft Matter 2012; 8:5974-5979.

19. Zhou ZL, Ngan AHW, Tang B, Wang AX. Reliable measurement of elastic modulus of cells by nanoindentation in an atomic force microscope. Journal of the Mechanical Behavior of Biomedical Materials 2012; 8:134-142.

20. Tang B, Ngan AHW. Nanoindentation using an atomic force microscope. Philosophical Magazine 2011; 91:1329-1338.

21. Guck J, Schinkinger S, Lincoln B, Wottawah F, Ebert S, Romeyke M, et al. Optical deformability as an inherent cell marker for testing malignant transformation and metastatic competence. Biophys $J$ 2005; 88:3689-3698.

22. Remmerbach TW, Wottawah F, Dietrich J, Lincoln B, Wittekind C, Guck J. Oral cancer 
diagnosis by mechanical phenotyping. Cancer Res 2009; 69:1728-1732.

23. Buckley ST, Davies AM, Ehrhardt C. Atomic force microscopy and high-content analysis: two innovative technologies for dissecting the relationship between epithelial-mesenchymal transition-related morphological and structural alterations and cell mechanical properties. Methods Mol Biol 2011; 784:197-208.

24. Rolli CG, Seufferlein T, Kemkemer R, Spatz JP. Impact of tumor cell cytoskeleton organization on invasiveness and migration: a microchannel-based approach. PLoS One 2010; 5:e8726.

25. Gerlitz G, Bustin M. The role of chromatin structure in cell migration. Trends Cell Biol 2011; 21:6-11.

26. Nakayama S, Sasaki A, Mese H, Alcalde RE, Matsumura T. Establishment of high and low metastasis cell lines derived from a human tongue squamous cell carcinoma. Invasion Metastasis 1998; 18:219-228.

27. Liu Z, Li S, Cai Y, Wang A, He Q, Zheng C, et al. Manganese superoxide dismutase induces migration and invasion of tongue squamous cell carcinoma via $\mathrm{H}(2) \mathrm{O}(2)$-dependent snail signaling. Free Radic Biol Med 2012;53:44-50.

28. Wang A, Zhang B, Huang H, Zhang L, Zeng D, Tao Q, et al. Suppression of local invasion of ameloblastoma by inhibition of matrix metalloproteinase-2 in vitro. BMC Cancer 2008; 8:182.

29. Ding X, Zhang N, Cai Y, Li S, Zheng C, Jin Y, et al. Down-regulation of tumor suppressor MTUS1/ATIP is associated with enhanced proliferation, poor differentiation and poor prognosis in oral tongue squamous cell carcinoma. Mol Oncol 2012; 6:73-80.

30. Nikkhah M, Strobl JS, De Vita R, Agah M. The cytoskeletal organization of breast carcinoma and fibroblast cells inside three dimensional (3-D) isotropic silicon microstructures. Biomaterials 2010; 31:4552-4561.

31. White FH, Jin Y, Yang L. An evaluation of the role of nuclear cytoplasmic ratios and nuclear volume densities as diagnostic indicators in metaplastic,dysplastic and neoplastic lesions of the human cheek. Histol Histopathol 1997; 12:69-77. 
32. Acerbi I, Luque T, Gimenez A, Puig M, Reguart N, Farre R, et al. Integrin-Specific Mechanoresponses to Compression and Extension Probed by Cylindrical Flat-Ended AFM Tips in Lung Cells. PLoS ONE 2013; 7: e32261.

33. Morita Y, Mukai T, Ju Y, Watanabe S. Evaluation of Stem Cell-to-Tenocyte Differentiation By Atomic Force Microscopy to Measure Cellular Elastic Moduli. Cell Biochem Biophys 2012; DOI 10.1007/s12013-012-9455-x

34. Mackay JL, Kumar S. Measuring the elastic properties of living cells with atomic force microscopy indentation. Methods Mol Biol 2013;931:313-329.

35. Xu W, Mezencev R, Kim B, Wang L, McDonald J, Sulchek T. Cell Stiffness Is a Biomarker of the Metastatic Potential of Ovarian Cancer Cells. PLoS ONE; 7: e46609.

36. Ngan AHW, Tang B. Response of power-law-viscoelastic and time-dependent materials to rate jumps. Journal of Materials Research 2009; 24:853-862.

37. Tang B, Ngan AHW. Nanoindentation measurement of mechanical properties of soft solid covered by a thin liquid film. Soft Materials 2007; 5:169-181.

38. Tang B, Ngan AHW, Lu WW. An improved method for the measurement of mechanical properties of bone by nanoindentation. Journal of Materials Science-Materials in Medicine 2007; 18:1875-1881.

39. Chan BP, Li CH, Au-Yeung KL, Sze KY, Ngan AHW. A microplate compression method for elastic modulus measurement of soft and viscoelastic collagen microspheres. Annals of Biomedical Engineering 2008; 36:1254-1267.

40. Chan YL, Ngan AHW. Invariant elastic modulus of viscoelastic materials measured by rate-jump tests. Polym Test 2010; 29:558-564.

41. Fuhrmann A, Staunton JR, Nandakumar V, Banyai N, Davies PC, Ros R. AFM stiffness nanotomography of normal, metaplastic and dysplastic human esophageal cells. Phys Biol 2011; 8:015007.

42. Ketene AN, Schmelz EM, Roberts PC, Agah M. The effects of cancer progression on the viscoelasticity of ovarian cell cytoskeleton structures. Nanomedicine 2012; 8: 93-102. 
43. Sakamoto K, Imanishi Y, Tomita T, Shimoda M, Kameyama K, Shibata K, et al. Overexpression of SIP1 and Downregulation of E-cadherin Predict Delayed Neck Metastasis in Stage I/II Oral Tongue Squamous Cell Carcinoma After Partial Glossectomy. Ann Surg Oncol 2012; 19: $612-619$.

44. Buckley ST, Medina C, Davies AM, Ehrhardt C. Cytoskeletal re-arrangement in TGF-p1-induced alveolar epithelial-mesenchymal transition studied by atomic force microscopy and high-content analysis. Nanomedicine 2012;8:355-364.

45. Kokubo K, Igawa S, Fukuda A, Shinbo T, Hirose M, Masuda N, et al. The role of nitric oxide in reducing deformability of Lewis lung tumor cell stimulated by inflammatory cytokines. Nitric Oxide 2008; 19:312-319.

46. Cai X, Xing X, Cai J, Chen Q, Wu S, Huang F. Connection between biomechanics and cytoskeleton structure of lymphocyte and Jurkat cells: An AFM study. Micron 2010; 41:257-262.

47. Lee SY, Zaske AM, Novellino T, Danila D, Ferrari M, Conyers J, et al. Probing the mechanical properties of TNF-alpha stimulated endothelial cell with atomic force microscopy. Int $J$ Nanomedicine 2011; 6:179-195.

48. González Avalos P, Reichenzeller M, Eils R, Gladilin E. Probing compressibility of the nuclear interior in wild-type and lamin deficient cells using microscopic imaging and computational modeling. J Biomech 2011; 44:2642-2648.

49. Stroka KM, Aranda-Espinoza H. Effects of Morphology vs. Cell-Cell Interactions on Endothelial Cell Stiffness. Cell Mol Bioeng 2011; 4:9-27. 


\section{Figure legends}

\section{Figure 1 The elastic modulus values of TSCCs with different metastatic potentials}

A: Loading schedule for the rate jump method. When doing experiment, the tip was first retracted for $2 \mu \mathrm{m}$ within $1 \mathrm{~s}$, and then was made to approach the cells with a constant speed $0.55 \mu \mathrm{m} / \mathrm{s}$ for $10 \mathrm{~s}$ and then held for 30s. After that, the tip was retracted with a constant speed $0.1 \mu \mathrm{m} / \mathrm{s}$ for $10 \mathrm{~s}$. The final unload point at $41 \mathrm{~s}$ was treated as the rate-jump point where eqn. (2) was applied.

B: The indentation force $v s$ time curve in rate jump tests using the loading profiles in Fig. 1A.

C: The elastic modulus of TSCC cells. Inside each box chart, the body denotes the values of $90 \%$ tested samples in the region, the number on top of each box chart is the mean value for each group, which is also the line inside the body box. The two ends of each box chart are the maximum and minimum value. Significant differences were found between Primary cells obtained from Case 3 and primary cells obtained from the other three cases; UM1 cells and UM2 cells; UM1/Control cells and $\mathrm{UM} 1 / \mathrm{miR}-138$ cells $(P<0.05)$.

\section{Figure 2 The metastatic potential of TSCC cells}

The migration ability of TSCC cells was assessed using a wound healing assay (A, B, C). The invasion ability of TSCC cells was assessed using a transwell invasion assay (D, E, and F). UM1 cells, UM1/Control cells and primary cells obtained from Case 3 had a higher migratory and invasive ability than UM2 cells, UM1/miR-138 cells and primary cells obtained from the other three cases, respectively. $*: P<0.05$.

\section{Figure 3 The morphology of TSCCs with different elastic modulus}

The cell morphology of TSCC primary cells and cell lines were detected under SEM as described in the Materials and Methods section. Primary cells obtained from Case 3, UM1 cells and UM1/Control cells had a fibroblastic morphology. Primary cells obtained from the other three cases, UM2 cells and UM1/miR-138 cells had a more adherent phenotype, which promoted the formation of cell clusters. $\longrightarrow$ : microvilli; $\cdots \cdots$ : filopodia-like processes. 


\section{Figure 4. The expression of EMT markers in TSCCs with different elastic modulus}

The EMT markers E-cadherin and vimentin were detected as described in the Materials and Methods section. Using immunohistochemistry (A, B), Primary cells obtained from Case 3 (in both cancer and LN tissue) had a higher expression level of vimentin and a lower expression level of E-cadherin compared with primary cells obtained from the other three cases, $P<0.05$. Semi-quantitative analysis of the immunohistochemistry is shown in B. Relative staining intensities were analyzed by using Image Pro Plus 6 software. Western blot analysis (C, D) demonstrated a higher expression level of vimentin and a lower expression level of E-cadherin in UM1 cells compared to UM2 cells (C), or UM1/Control cells compared to UM1/miR-138 cells (D).

\section{Figure 5 F-actin in TSCCs with different elastic modulus}

F-actin was detected with immunofluorescence as described in the Materials and Methods section.

The F-actin cytoskeleton features a dense cortical layer underneath the plasma membrane and an isotropic network throughout the cell body. Nuclear staining showed that the shadows within the cells coincide with the nucleus (not included in the image). F-actin intensity was significantly decreased in the cells with lower elastic modulus [i.e., primary cells obtained from Case 3, UM1 cells and UM1/Control cells] compared with cells with higher elastic modulus [i.e., primary cells obtained from the other three cases, UM2 cells and UM1/miR-138 cells], respectively. $P<0.05$.

\section{Figure $6 \beta$-tubulin in TSCCs with different elastic modulus}

$\beta$-tubulin was detected with immunofluorescence as described in the Materials and Methods section. The $\beta$-tubulin was localized in the space between the nucleus and the cell membrane. Nuclear staining showed that the shadows within the cells coincide with the nucleus (not included in the image). $\beta$-tubulin intensity were significantly increased in primary cells obtained from the other three cases, UM2 cells and UM1/miR-138 cells compared with primary cells obtained from Case 3, UM1 cells and UM1/Control cells, respectively. $P<0.05$. 
Table S1 Clinical characteristics of four TSCC cases

\begin{tabular}{lccccccc}
\hline & & Age & & Pathology & LN & \\
Case & Gender & (ys) & TNM & diagnosis & metastasis & Recurrence & Follow-up \\
\hline 1 & male & 53 & T3N0M0 & Middle-D & N & N & $18 \mathrm{~m}$ \\
2 & female & 37 & T3N0M0 & High-D & N & N & $12 \mathrm{~m}$ \\
3 & male & 59 & T2N1bM0 & Middle-D & Y & $1 \mathrm{~m} \mathrm{ps}$ & die $6 \mathrm{~m} \mathrm{ps}$ \\
4 & male & 59 & T1N0M0 & Middle-D & N & N & $10 \mathrm{~m}$ \\
\hline
\end{tabular}

*: ys: years; ps: post-surgery; D: diffententiation; Y: yes; N: no; m: months 
Table S2 Elastic modulus and nucleus size of $1386 \mathrm{Ln} / \mathrm{Control}$ cells and $1386 \mathrm{Ln} / \mathrm{miR}-138$ cells

\begin{tabular}{llll}
\hline & nucleus size $\left(\mu \mathrm{m}^{2}\right)$ & $\mathrm{N} / \mathrm{C}$ ratio & $\mathrm{E}_{\text {elastic }}(\mathrm{kPa})$ \\
\hline 1386Ln/Control $(\mathrm{n}=38)$ & $178.19 \pm 48.17^{*}$ & $0.39 \pm 0.05^{*}$ & $4.51 \pm 1.14^{*}$ \\
& & & \\
$1386 \mathrm{Ln} / \mathrm{miR}-138(\mathrm{n}=59)$ & $207.30 \pm 78.34$ & $0.25 \pm 0.03$ & $6.77 \pm 1.50$
\end{tabular}

The data are presented as mean \pm standard deviation. *: compared between 1386Ln/Control cells and 1386Ln/miR-138 cells, $P<0.05$. 
Table S3 The association between elastic modulus/migratory and elastic modulus/invasion

\begin{tabular}{lllllll} 
& & \multicolumn{2}{c}{ Pearson } & \multicolumn{2}{c}{ Spearman } \\
& & r & $p$ value & r & $p$ value \\
\hline Elastic modulus/relative migration & & TSCC cell lines & -0.688 & 0.000 & -0.677 & 0.000 \\
& TSCC cases & -0.682 & 0.000 & -0.782 & 0.000 \\
Elastic modulus/relative Invasion & TSCC cell lines & -0.639 & 0.000 & -0.632 & 0.000 \\
& TSCC cases & -0.720 & 0.000 & $-0.782^{*}$ & 0.000
\end{tabular}

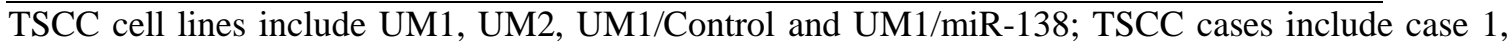
case 2, case 3 and case 4 . The TSCC cell lines and cases were classified by their migration and invasion (shown in Figure 2 and Figure S2), with the migration and invasion value of UM2 cells or case 1 falling into 1 , and the migration and invasion value of other TSCC cell lines and cases was descibed in comparision with UM2 cells or case 1, respectively. 


\section{Supplementary figure legends:}

Figure S1 TSCC cells were mechanically probed by AFM at their most bulging part

Figure S2 The migration and invasion ability of another paired OSCC cell lines (1386LN/Control and 1386LN/miR-138)

The migration ability was assessed using a wound healing assay (A). The invasion ability was assessed using a transwell invasion (B). 1386LN/Control cells had a higher migratory and invasive ability than $1386 \mathrm{LN} / \mathrm{miR}-138$ cells. *: $P<0.05$.

Figure S3 Histograms of elastic modulus for each sample from TSCC cell lines and patients

Figure S4 Morphology and cytoskeleton of another paired OSCC cell lines (1386LN/Control and 1386LN/miR-138)

A: The morphology of $1386 \mathrm{Ln} /$ Control and 1386Ln/miR-138 cells under SEM. 1386Ln/Control cells appeared spread out assuming a fibroblastic morphology. 1386Ln/miR-138 cells appeared more adherent to one another and promoted formation of cell clusters.

B: Cell cytoskeleton F-actin and $\beta$-tubulin in $1386 \mathrm{Ln} / \mathrm{Control}$ and $1386 \mathrm{Ln} / \mathrm{miR}-138$. The F-actin cytoskeleton features a dense cortical layer underneath the plasma membrane and an isotropic network throughout the cell body. The F-actin stress fiber was found to reduce and less organize in higher metastatic potential $1386 \mathrm{Ln} /$ Control as compared to $1386 \mathrm{Ln} / \mathrm{miR}-138$. *: $P<0.05$. The $\beta$-tubulin was localized in the space between nucleus and cell membrane, the $\beta$-tubulin was found to increase and more organize (around nucleus) in 1386Ln/miR-138 as compared to $1386 \mathrm{Ln} / \mathrm{Control}$. *: $P<0.05$. 
Fig. 1

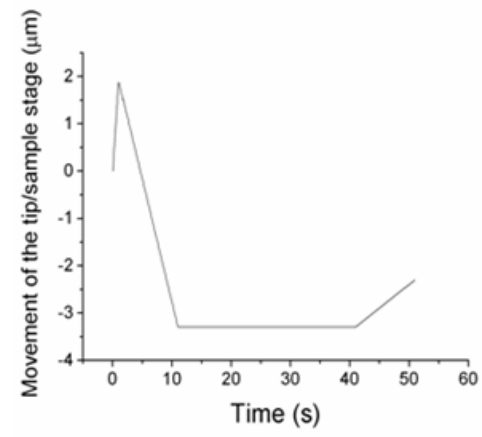

A

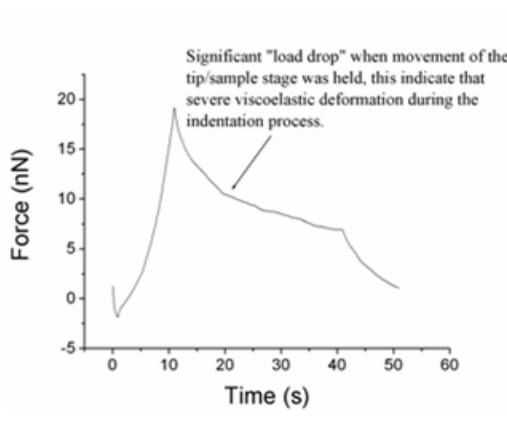

B

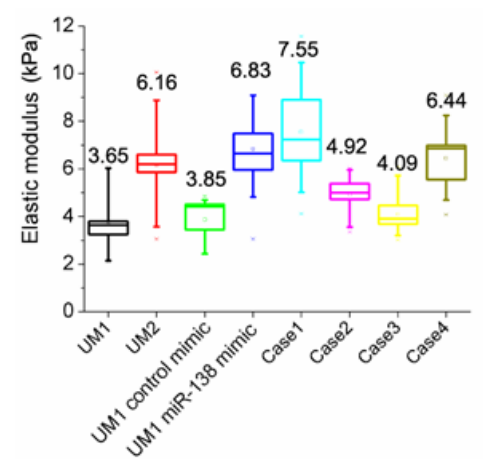

C

Fig. 2
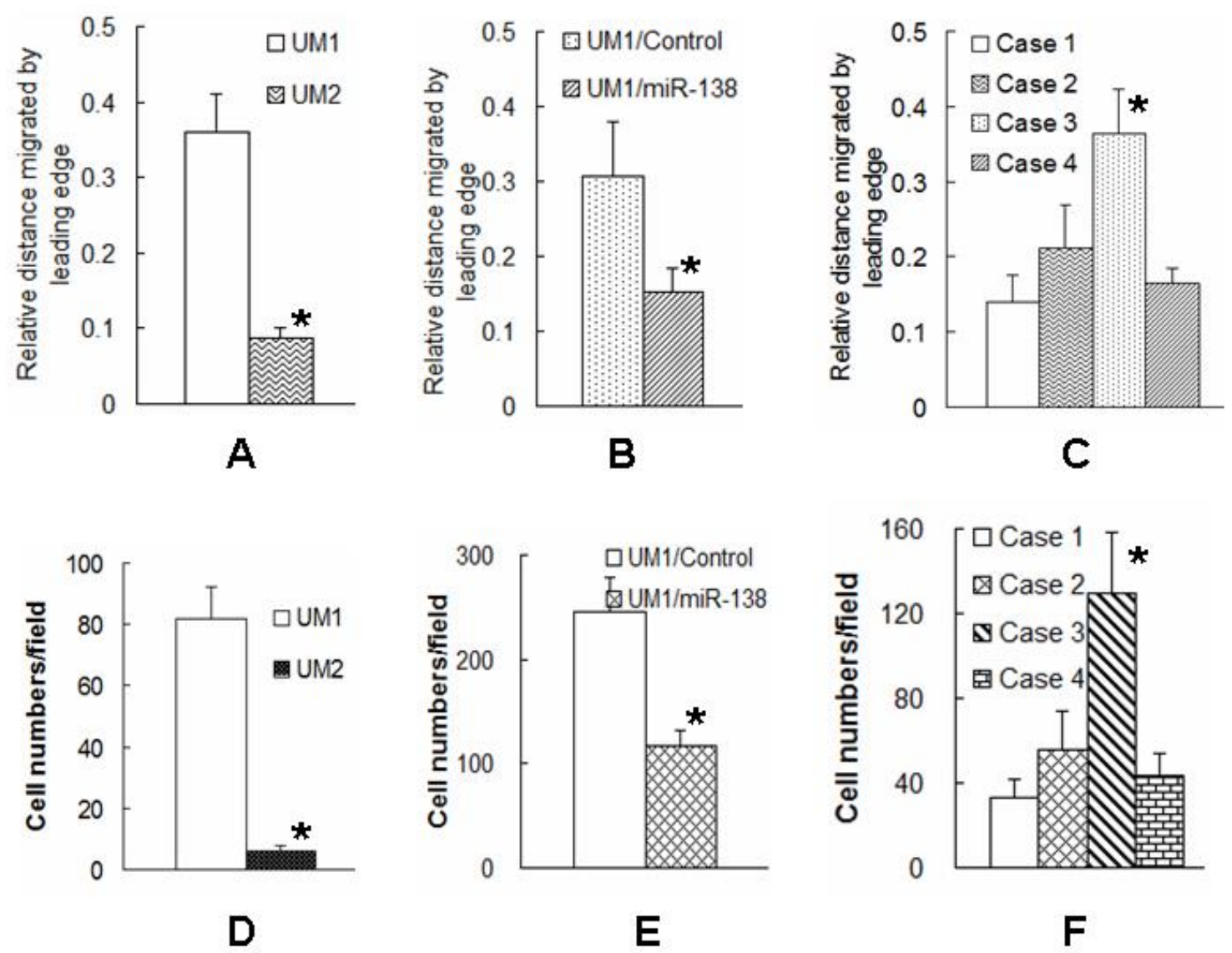
Fig. 3
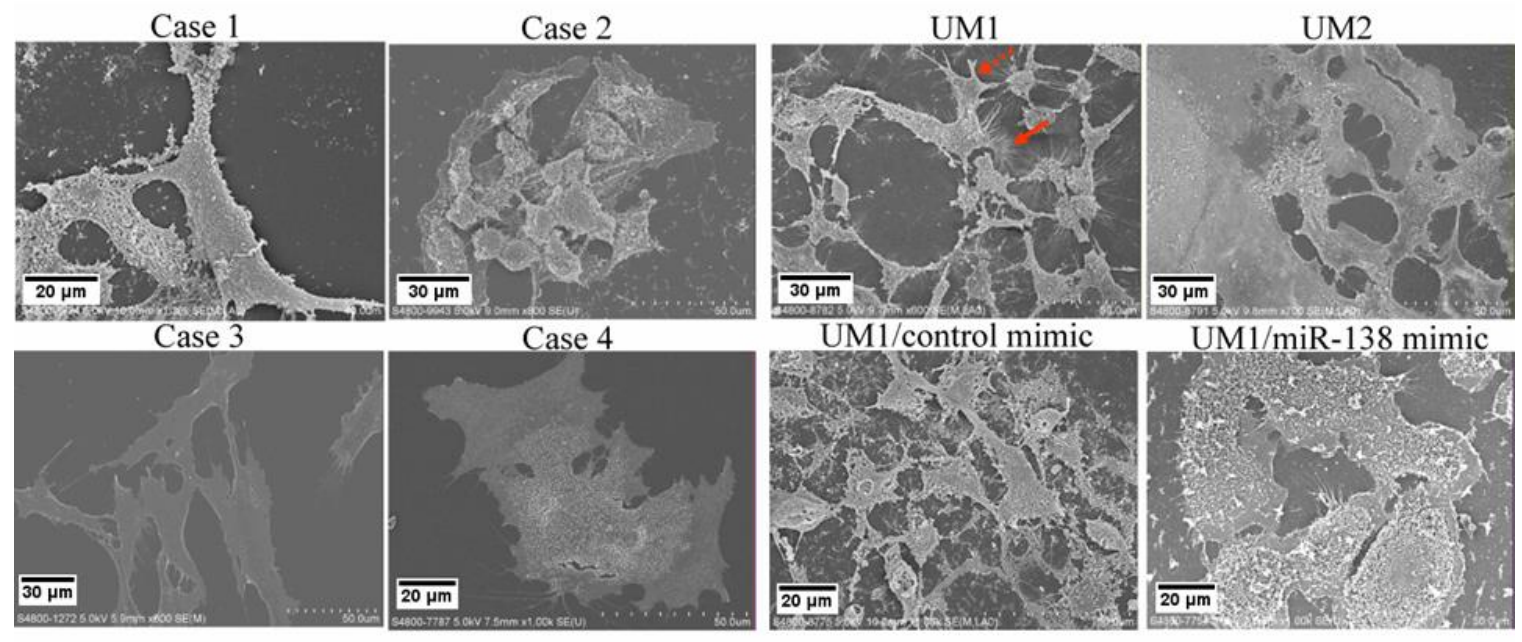


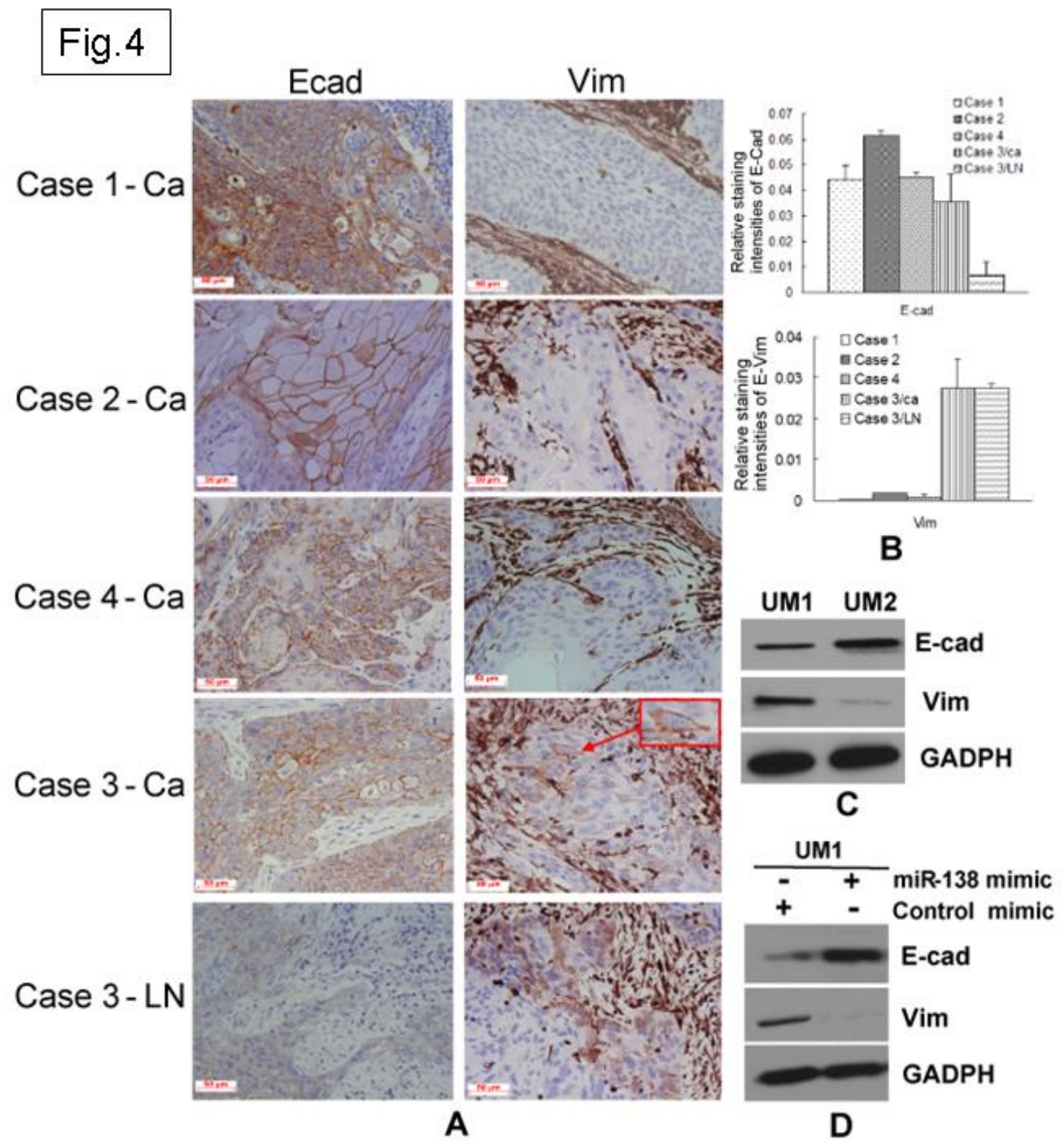




\section{Fig. 5}

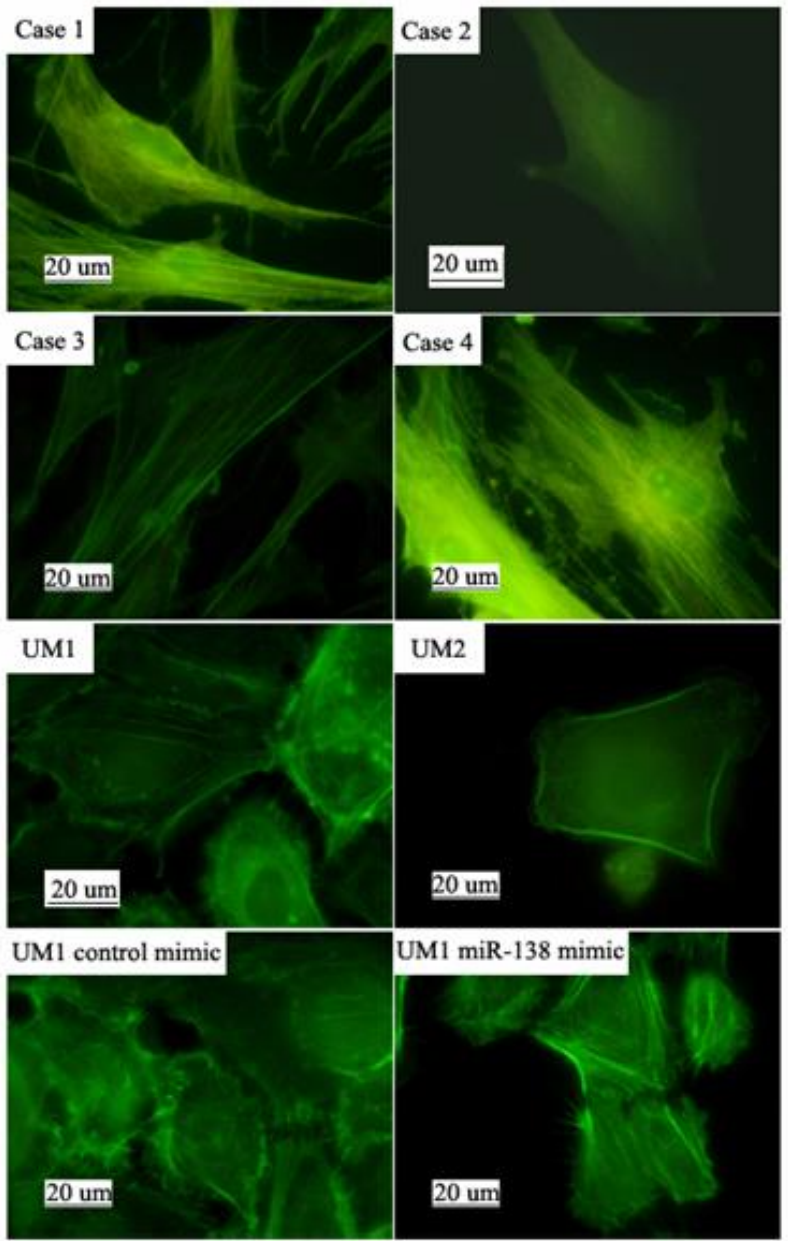

A

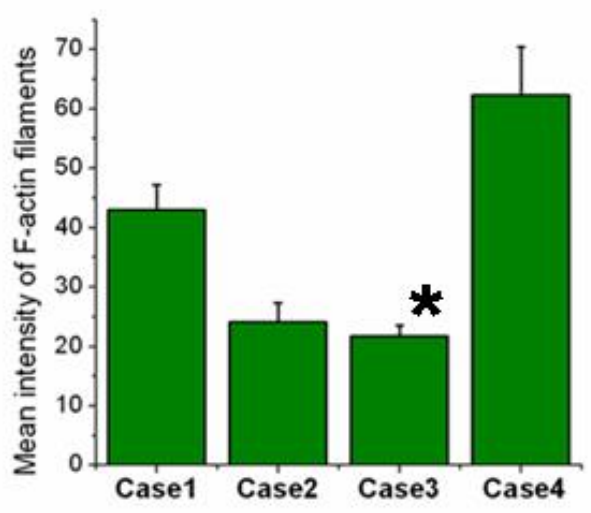

B

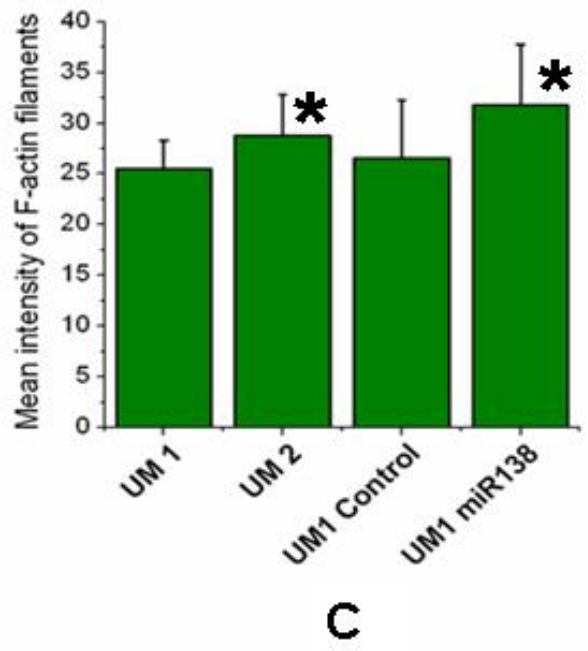


Fig.6

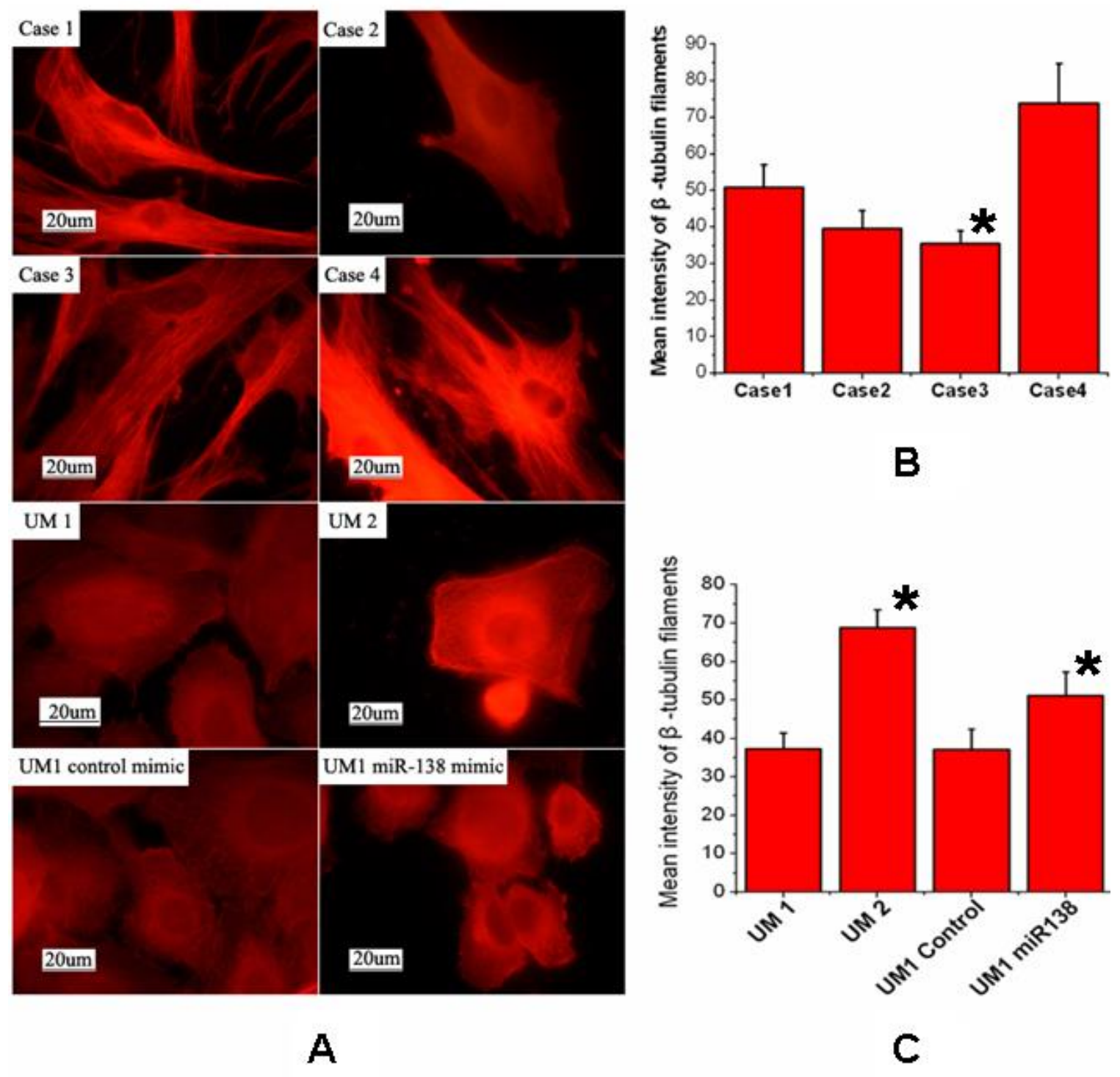


Fig.S1

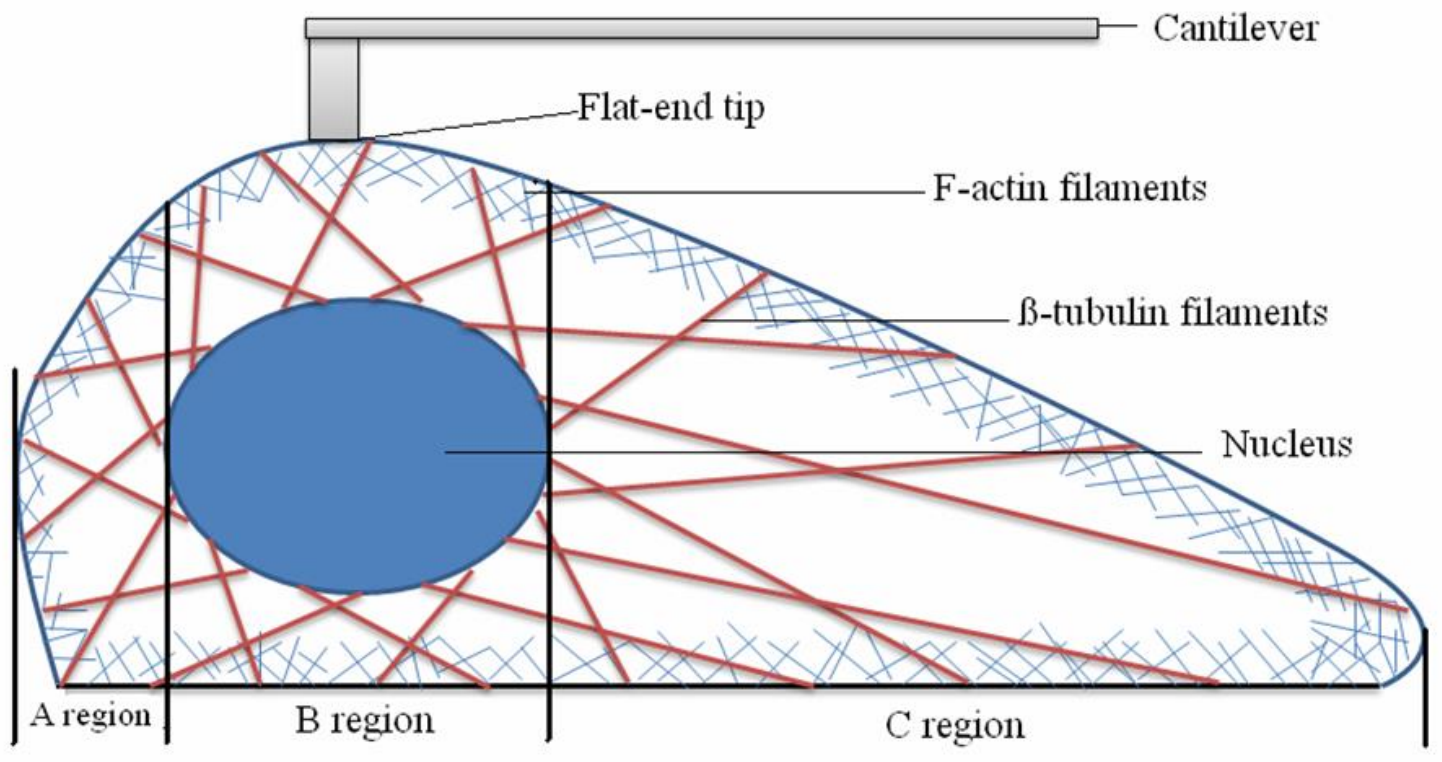

Fig.S2
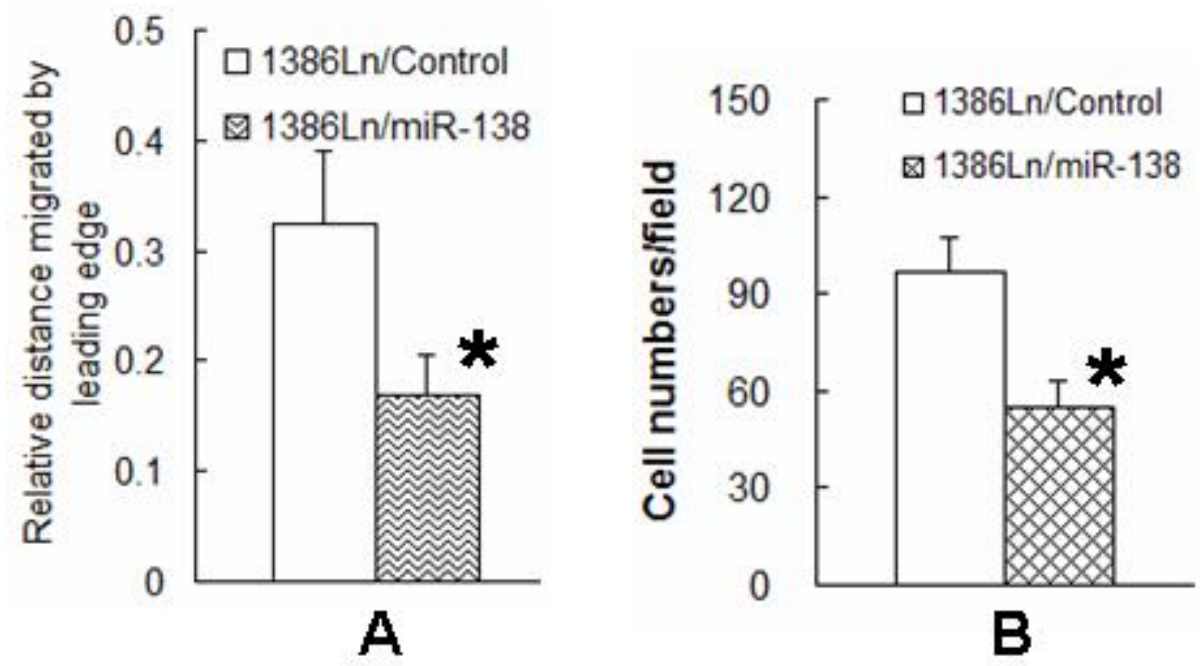
Fig.S3

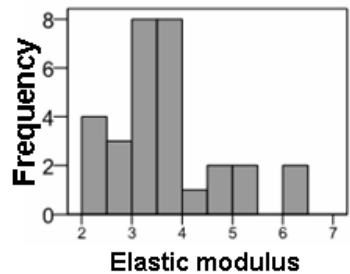

UM1

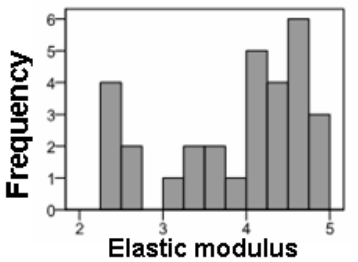

UM1/Control

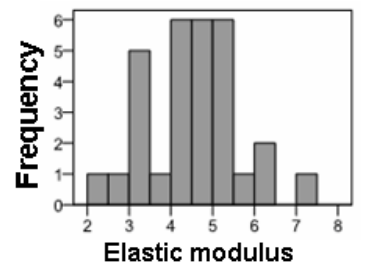

1386LN/Control

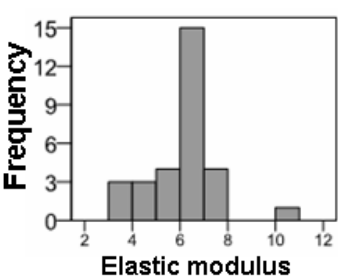

UM2

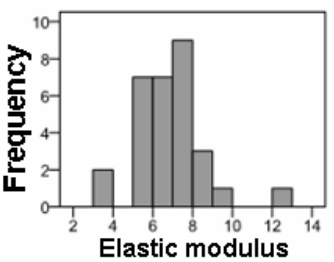

UM1/miR-138

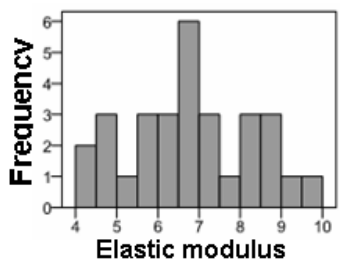

1386LN/miR-138
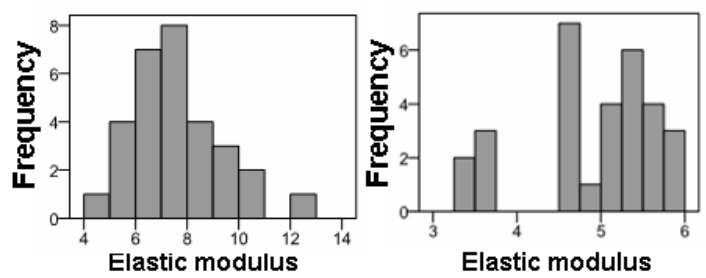

Case 1

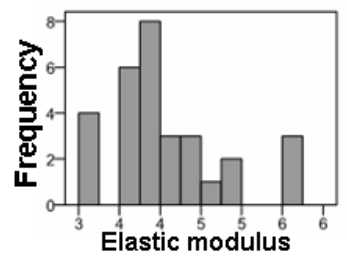

Case 3
Case 2

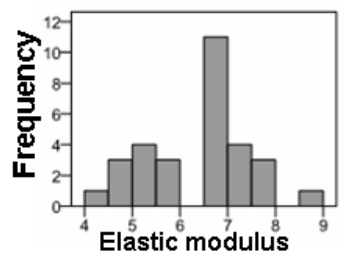

Case 4 
Fig. 54
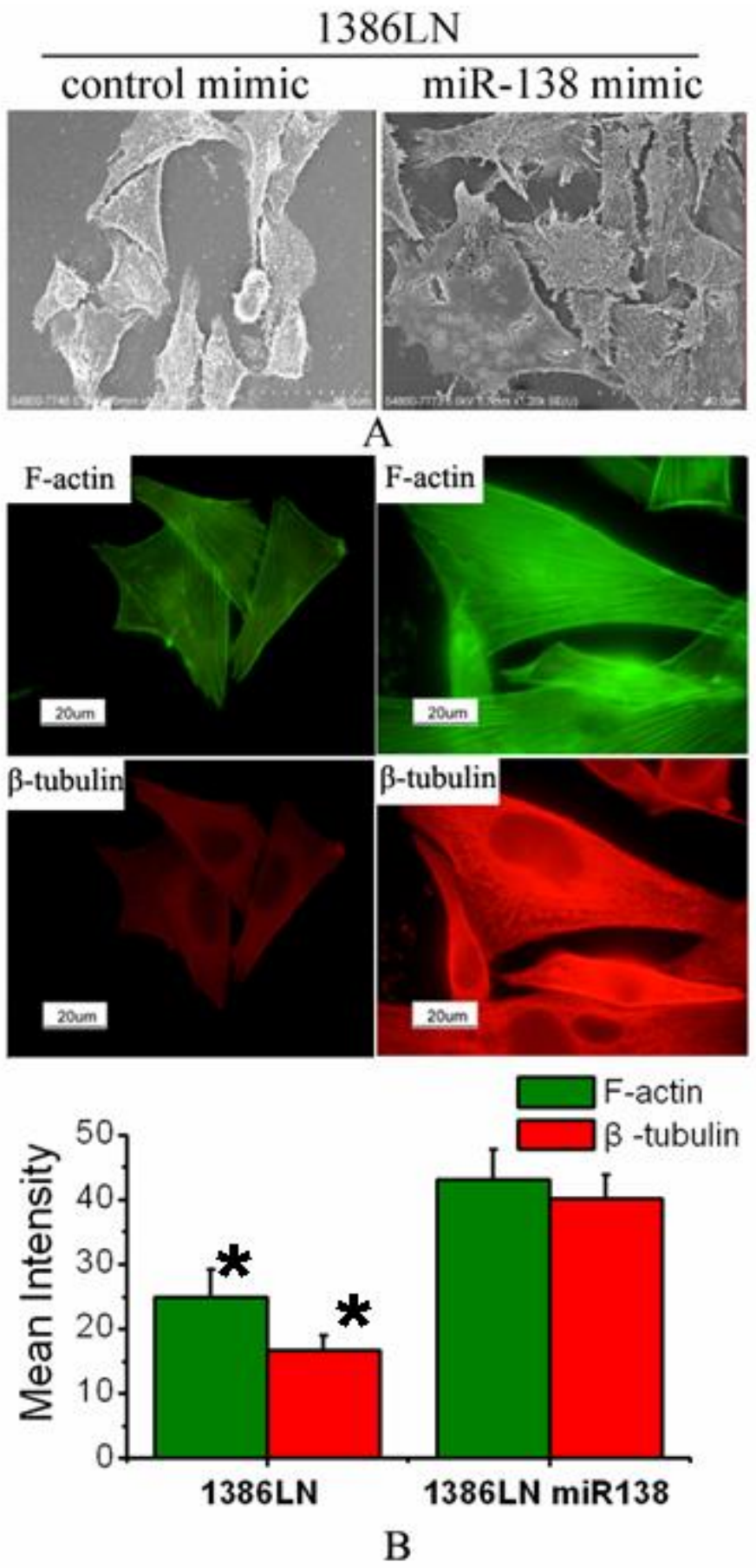